\title{
Discussion of Relationship between Hematuria and Ultrasonic Renal Calculi in Young Man After Exercise
}

\author{
Jian Luo*, Yue Zhao and Bo Zhou \\ Changsha Center for Disease Control and Prevention, Changsha, Hunan 410000, China
}

\begin{abstract}
Objective: To discuss the relationship between hematuria and ultrasonic renal calculi in young man after exercise. Method: In the medical observation, 48 cases of young male patients with hematuria after exercise in our hospital from January 2013 to December 2014 were selected as the observation objects, the subjects were transient hematuria, and 92 patients without hematuria were selected as the contrast, all patients underwent ultrasound examination, the detection rate of renal calculi and the ultrasonic manifestation were retrospectively analyzed. Results: The incidence of renal calculi of the young and middle-aged male patients with hematuria after exercise is statistically different in different parts and different age $(\rho<0.05)$, and the incidence rate of renal calculi in patients with hematuria after exercise and patients without hematuria was significantly different $(\rho<0.05)$, as shown in Table 1 , Table 2 . There was no obvious linear relationship between the degree of hematuria and the incidence of renal calculi after young men's exercise, as shown in Table 3. Conclusion: From this clinical research results we can know that renal calculi is the main cause of hematuria after exercise for the young man, and the clinical examination and diagnosis of renal calculi with ultrasonography has higher clinical detection rate, therefore the clinical application value is higher.
\end{abstract}

\author{
KEYWORDS \\ Young man \\ Hematuria after exercise \\ Ultrasonography \\ Renal calculi
}

\section{Introduction}

The clinical research analyzed the relationship between hematuria after exercise of young man and ultrasound renal calculi, the results was reported as follows.

\section{Materials and methods}

\subsection{Clinical data}

In the medical observation, 48 cases of young male patients

Copyright $\odot 2015$ Jian Luo et al.

doi: $10.18686 /$ aem.v4i3.2

Received: August 8, 2015; Accepted: September 3, 2015; Published online: September 17, 2015

This is an open-access article distributed under the terms of the Creative Commons Attribution Unported License (http://creativecommons.org/ licenses/by-nc/4.0/), which permits unrestricted use, distribution, and reproduction in any medium, provided the original work is properly cited.

${ }^{\star}$ Corresponding author: Changsha Center for Disease Control and Prevention, Changsha, Hunan 410000, China.

E-mail: luoj1989@163.com with hematuria after exercise in our hospital from January 2013 to December 2014 were selected as the observation objects, 92 cases of patients without hematuria were randomly selected as the control group, the age range of observation objects is between 18 to 39 years old, the average age was $(27.5 \pm 7.5)$ years old. All the observation objects had obvious symptoms of hematuria after exercise.

\subsection{Method}

The clinical study selected YD-URISCANS300 urine analyzer and the corresponding dry chemistry test paper (South Korea Yingdong Pharmaceutical Company) as the clinical examination equipment, inspection was made in strict accordance with the relevant instructions and the 11 items of urinary detection and instrument calibration method developed by 1995 Chinese society of laboratory Wuyi conference and international Committee for cell standard (NCCLS) [1].

(1) Sports test method: According to the different 
Table 1. The occurrence of hematuria of renal calculi patients with different ages (case\%).

\begin{tabular}{ccccc} 
& $\begin{array}{c}\text { Renal calculi and hematuria } \\
\text { positive group }\end{array}$ & $\begin{array}{c}\text { Renal calculi and non-hematuria } \\
\text { group }\end{array}$ & $\mathrm{X}^{2}$ & $\mathrm{P}$ \\
\hline $18-29$ years old & $45.71(16 / 35)$ & $11.11(7 / 54)$ & 11.886 & $=0.001$ \\
30-39 years old & $53.84(7 / 13)$ & $13.15(5 / 38)$ & 8.916 & $=0.006$ \\
Total & $47.92(23 / 48)$ & $13.04(1292)$ & 20.459 & $<0.001$ \\
\hline
\end{tabular}

Table 2. The occurrence of hematuria of patients with renal calculi in different parts (case\%).

\begin{tabular}{|c|c|c|c|c|c|c|c|c|}
\hline \multicolumn{2}{|c|}{ Double kidney stone } & \multicolumn{3}{|c|}{ Right kidney stone } & \multicolumn{2}{|c|}{ Left kidney stone } & \multirow{2}{*}{$\begin{array}{c}\mathrm{X} 2 \\
\begin{array}{l}\text { Right kidney and double } \\
\text { kidney } 0.329\end{array}\end{array}$} & \multirow{2}{*}{$\begin{array}{c}P \\
0.566\end{array}$} \\
\hline Hematuria & $\begin{array}{c}\text { Non } \\
\text { hematuria }\end{array}$ & Hematuria & Non & hematuria & Hematuria & Non hematuria & & \\
\hline $70(7 / 10)$ & $30(3 / 10)$ & $80 \quad(12 / 15)$ & 20 & $(3 / 15)$ & $40(4 / 10)$ & $60(6 / 10)$ & $\begin{array}{l}\text { Right kidney and left kidney } \\
4.199\end{array}$ & 0.040 \\
\hline / & / & / & & / & / & / & $\begin{array}{l}\text { Left kidney and double kidney } \\
1.818\end{array}$ & 0.178 \\
\hline
\end{tabular}

Table 3. The relationship between hematuria degree and the occurrence of renal calculi (case\%).

\begin{tabular}{cccccc}
\hline Microscopic hematuria & $++++\sim+++$ HP & $++\sim+$ HP & 0 9/HP & $X^{2}$ \\
\hline Renal calculus & $71.43(5 / 7)$ & $50.00(7 / 14)$ & $40.74(11 / 27)$ & 2.132 & 0.344 \\
Non renal calculi & $28.57(2 / 7)$ & $50.00(7 / 14)$ & $59.26(16 / 27)$ & \\
\hline
\end{tabular}

psychological quality of patients, choose the corresponding outdoor sports, mainly include: Jumping obstacles, running pass, basketball game, rope skipping, chasing, varied direction running, $25 \mathrm{~m}$ shuttle running, hand exercises, each movement lasts for $1 \mathrm{~h}$. After exercise stop for $30 \mathrm{~min}, 30 \mathrm{~mL}$ urine samples were collected, all urine samples receive microscopic examination and dry chemical detection simultaneously [2].

(2) Morning urine examination method: All observation objects collect $30 \mathrm{ml}$ urine in the morning, 30 min later, implement 11 items of dry chemical examination, of which the observation object with urine $\mathrm{RBC}( \pm)$ or above need to promptly receive centrifugation microscope examination [3].

(3) Urine sediment microscopy method: All patients need to collect $10 \mathrm{~mL}$ urine, centrifuge under $2000 \mathrm{rpm}$ for more than $3 \mathrm{~min}$. Collect $0.5 \mathrm{~mL}$ urine sediment, after smear mixing, examination of 10 fields is made under high power microscope, and calculate the average value. For patients with proteinuria dry chemical test results of $( \pm)$ or more, correction should be made by acetic acid heating method [4].

TOSHIBA SSA-550A ultrasonic diagnostic apparatus and SONOLINE G20 ultrasonic diagnostic apparatus are used in the examination, all the renal calculi are confirmed by CT scan examination, X-ray images and renal angiography.

\subsection{Statistical processing}

Data were analyzed by SPSS 17.0 software. ( $x \pm s$ ) was used to represent the measurement data, the single factor analysis of variance was used to compare the data, $\mathrm{X}^{2}$ test was used for statistical analysis of count data, if $p<0.05$, the difference between the data was statistically significant [5].

\section{Results}

The incidence of renal calculi in different parts of the young man with different age had statistical difference $(p<0.05)$, and the incidence rate of renal calculi in patients with hematuria after exercise and patients without hematuria was significantly different $(p<0.05)$, as shown in Table 1 and Table 2. There was no obvious linear relationship between the degree of hematuria and the incidence of renal calculi after young men's exercise, as shown in Table 3.

\section{Discussion}

Hematuria of young man after exercise is a common renal disease in clinical, the occurrence rate is next only to nephritic syndrome and nephritis due to acute streptococcus infection, accurate and reliable diagnosis is usually made through renal biopsy, imagological diagnosis, blood and urine examination, physical inspection, clinical historical survey and other system examination. According to the source of urinary red blood cells, it can be divided into two types: non renal hematuria and renal hematuria. The main source of urinary red blood cells of former type is from other urinary system organs except for glomerulus, and the source of latter type is usually glomerulus.

Related medical research results confirmed that different degree of hematuria will occur in about $92 \%$ of young men after high intensity exercise, the main factors include the following: first, after movement, the bladder will remain at empty state, bladder wall will encounter repeated impact, 
so it is easy to cause hematuria resulted from bladder injury. Second, after high intensity exercise, the urinary system will have a strong vibration, which will lead to the occurrence of large renal upper and lower movement, on the other hand, affected by the waist extension and flexion and other factors, the kidney will be squeezed, and then twist or stretch renal blood vessels, leading to renal injury bleeding. Third, after high intensity exercise, the kidney will experienced carbon dioxide retention, renal tissue hypoxia ischemia, renal blood flow reduction and renal blood vessel contraction and other phenomenon, thereby leading to hematuria.

Renal calculi refer to the stones occur in the renal calices, the renal pelvis and the connection part of renal pelvis and the ureter. Plain radiographs showed single or multiple round, oval or blunt triangular dense image in renal area, the density is high and uniformity. The edge usually is smooth, but there is also not a glossy diverge. Kidney is the main site of urinary system stones, any stones of other parts can originate from kidney, ureteral stones are almost from the kidney, and renal calculi may more easily damage the kidney than calculi in any other part, so early diagnosis and treatment is very important. According to the different components, renal calculi can be divided into calcium oxalate stones, calcium phosphate stones, uric acid (uric acid salt) stones, magnesium ammonium phosphate stones, cysteine stone and purine stones.

The causes of renal calculi are more complicated, which usually include: drug interaction, anatomical factors, dietary factors, environmental factors, infectious factors, metabolic factors, genetic factors, etc. The clinical symptoms of renal calculi are more complex, the main symptom are fever. Bacterial infection is the main cause of renal calculi, so the patient is prone to fever symptoms; second are hydronephrosis. The occurrence of the stone will block the ureter and renal pelvis, and then affect the normal discharge of urine, which induce hydronephrosis. However, some patients are asymptomatic. Many patients with renal calculi did not have obvious clinical symptoms, and only discover by the physical examination, fourth symptom are including hematuria. The incidence of hematuria in patients with renal calculi is usually around $80 \%$.

Controlling of diet may be used to prevent renal calculi, or reduce the growth speed of renal calculi for the patient with renal calculi, even shrinking, dissolve and discharge it out from the body.

(1) Drink plenty of boiling water. Drinking plenty of water may dilute the urine, then decrease the concentration of calcium and oxalate, so that formation of calcium oxalate stones cannot occur. The study showed that the incidence of renal calculi was decreased by $86 \%$ with an increase of 50\% urine. (2) Reasoned calcium supplement, especially dietary calcium. Patients with renal calculi are often afraid of calcium, mistakenly believe that the culprit of renal calculi is calcium, actually otherwise, patient with nephrolithiasis and ureterolithiasis also need calcium. Two different perspectives from the current medical community explain that, first the consuming of calcium will react with oxalic acid contained from vegetables of gastrointestinal tract and form insoluble calcium oxalate, hence discharge out of body with feces, to reduce level of oxalic acid absorbed by the stomach and discharged by kidney, thereby reducing the risk of renal calculi formation. Second is the theory of acid-base balance proposed by Japanese scholars, namely when the blood is acidic, kidney stone easily form however in the alkaline condition it inhibit the formation of renal calculi. Lacking of calcium cause the blood become acidic, and reasonable calcium intake makes blood alkali, so that it is effective in the inhibition of kidney stones formation. (2) Limit sugar intake. The new study results of Scientists of the United States shows that the high sugar intake can increase the risks of renal calculi occurrence. Therefore, control our sugar intake. (4) Eat little foods with high oxalate. Foods with high oxalate are tomato, spinach, strawberries, sugar beet, chocolate and so on, high oxalate intake also results in renal calculi. (5) Eat less soy products. Soybean food contains high oxalate and phosphate, which can be integrated with calcium in the kidney to form stones. (6) Eat more black fungus. The black fungus is rich in various minerals and trace elements, can produce a strong chemical reaction to all kinds of stones, so that the stone is striped off, divided, dissolved and discharged.

In summary, ultrasonic examination has the advantages of repeatable, fast and convenient, simple, high repeatability, high accuracy, patients have small pain during the detection process, so it is the first choice for patients with renal calculi for clinical examination. All the patients with hematuria in clinical should receive ultrasonic examination of urinary system, to check renal calculi.

\section{Conflicts of interest}

These authors have no conflicts of interest to declare.

\section{Authors' contributions}

These authors contributed equally to this work.

\section{Reference:}

1. Chen XH, Song L, Song ZC. Application of tissue harmonic imaging technique in the diagnosis of renal calculi. Chinese Journal of Ultrasound Diagnosis. 2003;4(10):780781.

2. Sports medicine group of Hunan Medical College Affiliated Hospital Physical Therapy Department. Hematuria After exercise and motion hematuria. Chinese Journal of Internal Medicine. 1999;8(5):56-57.

3. Han SL, Liu J, Shi YL, et al. The affected factors of formation of calcium oxalate renal calculi and ultrasonic diagnosis value. Foreign medical geography volumes. 2005;26(2):94-95.

4. Fang JX. Diagnosis and Countermeasure of Adolescents hematuria after exercise. Sports health. 2010;1(1):61-62.

5. Jia J, Chen YH. Evaluation of the ultrasonic diagnosis value of hematuria without symptom. Journal of Tropical Medicine. 2002;2(4):409-410. 